\title{
2-Tuple Unbalanced Linguistic Multiple Criteria Group Decision Making using Prospect Theory Data Envelopment Analysis
}

Imran Khan ( $\boldsymbol{\sim}$ imranalig_khan@yahoo.co.in )

Rukmini Devi Institute of Advanced Studies https://orcid.org/0000-0002-2503-4552

\section{Anjana Gupta}

Delhi Technological University

\section{Aparna Mehra}

Indian Institute of Technology Delhi

\section{Research Article}

Keywords: multiple criteria group decision making, 2-tuple unbalanced linguistic terms, data envelopment analysis, prospect value function, cross-efficiency

Posted Date: August 3rd, 2021

DOl: https://doi.org/10.21203/rs.3.rs-594743/v1

License: (a) (1) This work is licensed under a Creative Commons Attribution 4.0 International License. Read Full License

Version of Record: A version of this preprint was published at Soft Computing on April 4th, 2022. See the published version at https://doi.org/10.1007/s00500-022-07039-2. 


\title{
2-Tuple Unbalanced Linguistic Multiple Criteria Group Decision Making using Prospect Theory Data Envelopment Analysis
}

\author{
Imran Khan ${ }^{\otimes} \cdot$ Anjana Gupta $\cdot$ Aparna Mehra
}

Received: date / Accepted: date

\begin{abstract}
The linguistic terms in a balanced linguistic term set describing qualitative data are symmetrical around the central linguistic word. With the growing complexity of the problems, the symmetric linguistic term set appears to be confined. This work examines the multiple criteria group decision-making problems where decision-makers employ a 2-tuple unbalanced linguistic term set to provide entries of alternativecriteria matrices. We adopt a data envelopment analysis (DEA) method and create a linear programming model to evaluate alternative-criteria weights for each decision-maker. The value function from prospect theory models the non-rational aspect of risk in criteria. The values of prospect gain and prospect loss on cost and benefit criteria are computed and used to create a DEA model that evaluates the weights of each criterion on each alternative. Finally, the entropy values of the cross-efficiency scores deliver a ranking of the alter-
\end{abstract}

I. Khan (corresponding author)

Rukmini Devi Institute of Advanced Studies, Rohini, New Delhi110085, India

E-mail: imranalig_khan@yahoo.co.in

\section{A. Gupta}

Department of Applied Mathematics, Delhi Technological University, Bawana Road, New Delhi-110042, India

E-mail: anjanagupta@dce.ac.in

\section{A. Mehra}

Department of Mathematics, Indian Institute of Technology Delhi, Hauz Khas, New Delhi-110016, India

E-mail: apmehra@maths.iitd.ac.in natives. A numerical example illustrates the proposed methodology.

Keywords multiple criteria group decision making - 2-tuple unbalanced linguistic terms - data envelopment analysis - prospect value function . cross-efficiency

\section{Introduction}

Multiple criteria decision-making (MCDM) problems get investigated in the literature for their wide range of applications in decision making, including supply chain management, medical diagnosis, alternative selection, technology evaluation, military system efficiency, and investment decision-making (see, Dursun and Karsak (2013); Ehrgott et al. (2004); Liu et al. (2015); Yue (2013); Yayla et al. (2015)), to cite a few.

An MCDM problem intends to rank a finite number of alternatives based on a limited number of criteria and the judgment of a single decision maker. A multiple criteria group decision-making (MCGDM) problem seeks the opinion of several decision-makers on an alternative-criteria system, with each decision maker providing the alternative-criteria matrices. The criteria get classified into two bins: cost (the lower is better) and benefit (the higher, the better)

In an MCGDM, all decision-makers assessments may not carry equal weight; based on the positional hierarchy, responsibility, expertise, and understanding, some 
experts may play more decisive roles than others in a group. As a result, determining the weights of the decision-makers in a group is critical. Furthermore, a decision maker may not be an authority on each criterion to assess an alternative accurately. If not always, the participating decision-makers often employ quantitative descriptors to communicate their assessments of alternatives on criteria.

In addition to the decision maker's expertise, the complexity of relationships among parameters contributes to uncertainty in evaluating alternatives. When dealing with unknown data, traditional numerical-based MCGDM modeling is insufficient. Researchers have effectively expanded the scope of MCGDM to include vagueness and complexity expressed by various tools. For instance, MCGDM problems are set up in a fuzzy environment using fuzzy sets, intuitionistic fuzzy sets, interval-valued sets, hesitant fuzzy sets, type-1 fuzzy sets, type-2 fuzzy sets, Pythagoras fuzzy sets to handle the uncertainties in information effectively. The literature is very elaborate to quote all; one can refer to Dong and Cooper (2016); Farhadinia and $\mathrm{Xu}$ (2020); Garg (2018); Sun et al. (2020) for more details on MCGDM using fuzzy sets and its variants. Furthermore, different MCGDM methods have been proposed and extensively applied to study problems in various domains. For example, analytical hierarchy process (AHP) (Büyüközkan et al. (2019); Wu and Tu (2021)), TOPSIS (Guo and Zhao (2015); Liang and Xu (2017); Papapostolou et al. (2017)), PROMETHEE (Qi et al. (2019)), VIKOR (Çalı and Balaman (2019); Li et al. (2020)), ELECTRE (Akram et al. (2020)), and a few others, including the amalgamation or hybrid of two or more methods like AHP-TOPSIS (Tian and Peng (2020)) or TOPSIS-VIKOR (Dammak et al. (2020)).

Herrera and Martínez (2000) put forward a 2-tuple linguistic computational model and developed a procedure of computing with words (CWW) on it. The CWW uses linguistic data to generate linguistic output through translation and mathematical operations. The 2-tuple linguistic model by Herrera and Martínez (2000) improves on the basic linguistic model by adding a parameter to enhance the linguistic correctness in computations after the re-translation process. Thus, un- like the conventional linguistic model, the domain of 2-tuple linguistic model is a continuous space, resulting in no loss of information in CWW. A balanced linguistic term set comprises uniform and symmetrical linguistic terms spread around the middle linguistic term. However, we run into a problem when evaluating variables involving language phrases that are not necessarily evenly and symmetrically distributed (Liu et al. (2004); Torra (2000)). We call it by an unbalanced linguistic term set (Herrera et al. (2008)). Recently, Malhotra and Gupta (2020a) proposed a minimum distance measure to construct a framework for CWW in unbalanced linguistic term set.

An MCGDM method involves three essential entities: decision-makers, criteria, and alternatives, and in essence, requires aggregating the information in the alternativecriteria matrices given by several decision-makers to arrive at a ranking of alternatives. In the aggregation process, the weights of decision-makers on each criterion and the weights of each alternative criterion are vital. In some studies (Ramanathan and Ganesh (1994); Saaty (1980)), the importance of decision-makers is prior given. In contrast, in certain other studies (like, Koksalmis and Kabak (2019); Yue (2013)), the decisionmakers' weights get determined using suitable techniques. Geng et al. (2017) employed the data envelopment analysis (DEA) approach to solve MCGDM problems integrating 2-tuple linguistic DEMATEL for capturing influence relationships among criteria. Wan et al. (2016) studied the MCGDM problem with intuitionistic fuzzy numbers and determined the decision maker's weights using TOPSIS. Dong and Cooper (2016) pointed out the significance of decision-makers weights in aggregation processes and used the Markov chain method to determine them. Ju (2014) applied the similarity degree in the 2-tuple linguistic evaluation and defined the 2-tuple linguistic positive, negative, and left negative ideal solutions and proposed optimization models to determine the weights of decisionmakers and criteria.

Against this brief background, the foremost objective of the present study is to provide a ranking of alternatives in an MCGDM problem when the decisionmakers use the 2-tuple unbalanced linguistic term set 
to supply the alternative-criteria matrices' entries. We use the DEA-based linear programming model to evaluate each decision maker's weights on each alternative criteria. These weights are applied to find the aggregate alternative-criteria matrix. The prospect theory is then used to decide the prospect gain and loss values on cost and benefit criteria of entries of the aggregated matrix and formulate a prospect values-based DEA model to determine each criterion's weights on each alternative. The alternatives get ranked using cross-efficiency scores and their entropy values.

DEA is a non-parametric methodology for determining relative efficiency of homogeneous decision making units (DMU) that use multiple inputs to produce multiple outputs. In the context of MCGDM, the alternatives act as DMUs. Farrell (1957) describes the notion of efficiency of a DMU with a single input and single output. Charnes et al. (1978) extended it to formulate an efficiency score for multiple inputs, multiple outputs case and introduced linear programming models to measure efficiency scores of DMUs. Since their pioneering work, DEA has come a long way in research and applications in various sectors such as energy, environment, investment, health care, manufacturing, and service industries, to state a few. Bouyssou (1999) studied the equivalence between efficiency in DEA and convex efficiency in MCDM. Ebrahimnejad et al. (2019) formulated a DEA model in a fuzzy environment and applied chance-constrained programming to solve such a DEA model.

Each DMU self-evaluates its best relative efficiency using its most favorable input and output weights from the DEA model. On the other hand, the crossefficiency method, proposed by Sexton et al. (1986) and refined by Doyle and Green (1994), analyzes the efficiency of a DMU not only by self-assessment but taking into account peer-evaluation also. The pear evaluation assesses each DMU with optimal weights of the other DMUs. The cross-efficiency score of a DMU is the average of its self-evaluated efficiency and its peerevaluated efficiencies.

Soleimani-Damaneh and Zarepisheh (2009) applied Shannon (1948) entropy to aggregate different efficiency results. Wu et al. (2012) introduced the entropy value of cross-efficiency score and developed a distance entropy function to generate relative weights for crossefficiency aggregation. Xie et al. (2014) used Shannon's entropy to derive degree of importance of each DMU and merged it with the efficiency score. A modified weight-restricted DEA model proposed by Qi and Guo (2014) using Shannon's entropy. Wang et al. (2016) used the DEA entropy model to determine cross-efficiency intervals with imprecise inputs and outputs.

The contributions of this paper can be highlighted as follows. Firstly, we provide a concise review of the linguistic MCGDM problem and propose a DEA linear program model to evaluate the weights of each decision-maker on each alternative-criteria. We used the power function of prospect theory to incorporate risk-averse perspective of cost criteria over the benefit criteria in computing the aggregated cross-efficiency scores. The entropy of cross-efficiency scores finally enable in ranking alternatives.

The remaining sections of the paper are designed as follows. Section 2 describes the unbalanced linguistic term set. Section 3 explains the DEA model for a MCGDM problem with alternative-criteria matrices entries from the unbalanced 2-tuple linguistic term set. Section 4 formulates the prospect theory-based DEA model to compute the cross-efficiency of alternatives. Section 5 describes the step-wise procedure to solve the underline MCGDM problem and ranking of the alternatives. Section 6 illustrates the proposed methodology with a numerical example. Section 7 concludes the paper.

\section{Unbalanced Linguistic Term Set}

The 2-tuple linguistic model adds a new parameter called symbolic translation to the basic fuzzy linguistic model. The model is represented by a pair $(l, \alpha)$, where $l \in L$ is a linguistic term from linguistic term set $L=\left\{l_{0}, l_{1}, \ldots, l_{g}\right\}$ and $\alpha \in[-0.5,0.5)$ is a numeric value assigned for symbolic translation. The 2-tuple linguistic model assists in explaining the process of CWW by imparting continuity to the linguistic domain. Martínez and Herrera (2012) and Malhotra and Gupta (2020b) presented the state-of-the-art reviews of the 2- 
tuple linguistic model and its applications to decisionmaking.

The multiplicative unbalanced 2-tuple linguistic term (MU2LT) set is introduced by Malhotra and Gupta (2020a).

Definition 1 Let $L S=\left\{t_{a^{-f}}, t_{a^{-(f-1)}}, \ldots, t_{a^{0}}, \ldots, t_{a^{8}}\right\}$ be a multiplicative unbalanced linguistic term (MULT) set with cardinality $f+g+1$, where $f, g$ are positive integers, and $a$ is a real number, $a>1$. Let $\beta \in\left[a^{-f}, a^{g}\right]$ be a numerical value representing the result of a symbolic aggregation. The function providing the unbalanced 2tuple linguistic value equivalence to $\beta$ is $\Omega:\left[a^{-f}, a^{g}\right] \rightarrow$ $L S \times[0.5,-0.5)$ defined by

$$
\Omega(\beta)=\left(t_{\lambda}, \gamma\right),
$$

where

$$
\begin{cases}\lambda=a^{\sigma_{L}} \text { or } \lambda=a^{\sigma_{R}}, & \sigma_{L}, \sigma_{R} \in\{-f, \ldots, 0, \ldots, g\}, \\ \gamma=\frac{\beta-\lambda}{d_{L}+d_{R}}, & \gamma \in[-0.5,0.5),\end{cases}
$$

and the following procedure Malhotra and Gupta (2020a) is used to compute $\sigma_{L}, \sigma_{R}, d_{L}$, and $d_{R}$.

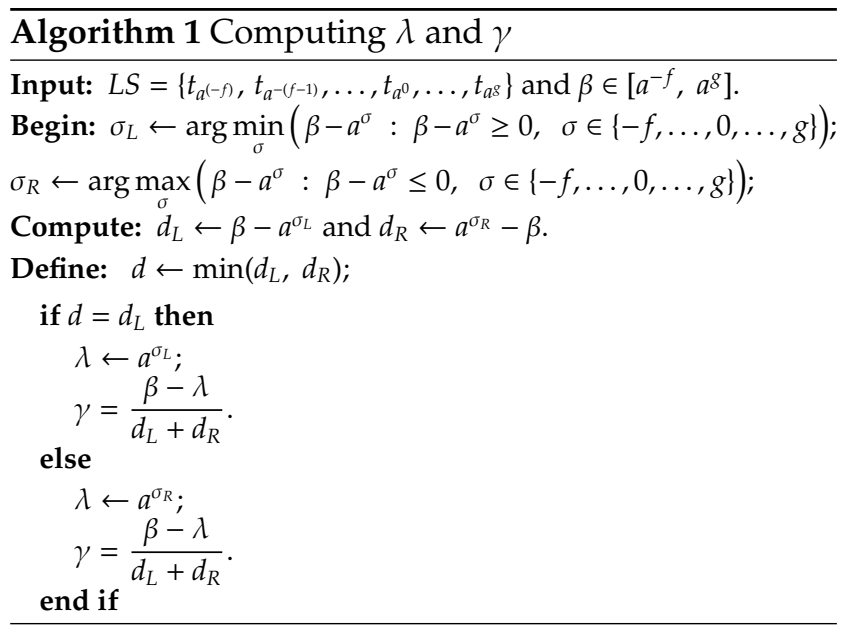

Definition 2 Let $L S=\left\{t_{a^{(-f)}}, t_{a^{-(f-1)}}, \ldots, t_{a^{0}}, \ldots, t_{a^{g}}\right\}$ be linguistic term set. The function $\Omega^{-1}: L S \times[-0.5,0.5) \rightarrow$ $\left[a^{-f}, a^{g}\right]$ transforms the unbalanced 2-tuple linguistic variable $\left(t_{\lambda}, \gamma\right)$ into its equivalent numerical value $\beta \in$ $\left[a^{-f}, a^{g}\right]$ by

$$
\Omega^{-1}\left(t_{\lambda}, \gamma\right)=\gamma\left(d_{L}+d_{R}\right)+\lambda
$$

Definition 3 Let ULS $=\left\{\left(t_{a^{\lambda_{1}}}, \gamma_{\lambda_{1}}\right), \ldots,\left(t_{a^{\lambda_{n}}}, \gamma_{\lambda_{n}}\right)\right\}$ be a set of $n$ multiplicative unbalanced 2-tuples, where $\lambda_{i} \in$ $\{-f,-(f-1), \ldots, 0, \ldots, g\}, i=1, \ldots, n$, and $t_{a^{\lambda}} \in L S$ and $\gamma_{\lambda} \in[-0.5,0.5)$. Let the weight vector be $W=$ $\left(w_{1}, \ldots, w_{n}\right)^{\prime}$, with $w_{i} \in(0,1)$, and $\sum_{i=1}^{n} w_{i}=1$. The weighted average operator (UWA) is defined as follows:

$$
\begin{array}{r}
U W A\left(\left(t_{a^{\lambda_{1}}}, \gamma_{\lambda_{1}}\right),\left(t_{a^{\lambda_{2}}}, \gamma_{\lambda_{2}}\right), \ldots,\left(t_{a^{\lambda_{n}}}, \gamma_{\lambda_{n}}\right)\right) \\
=\Omega\left(\sum_{i=1}^{n} w_{i} \Omega^{-1}\left(t_{a^{\lambda_{i}}}, \gamma_{\lambda_{i}}\right)\right) .
\end{array}
$$

\section{DEA Model for Aggregating Alternative- Criteria Matrices}

Let $\mathcal{A}=\left\{A_{1}, \ldots, A_{m}\right\}, m \geq 2$, and $C=\left\{C_{1}, \ldots, C_{n}\right\}$ be the sets of $m$ alternatives and $n$-criteria, respectively. Let there be $d$ decision-makers participating in a MCGDM problem, denoted by the set $\mathcal{D}=\left\{D M_{1}, D M_{2}, \ldots D M_{d}\right\}$, $d \geq 2$. Each decision maker expresses assessment of each alternative on each criterion in a linguistic description belonging to the set $L S=\left\{t_{a^{(-f)}}, t_{a^{-(f-1)}}, \ldots, t_{a^{0}}, \ldots, t_{a^{8}}\right\}$. Let the alternative-criteria matrix of the $k^{\text {th }}$ decision maker be $\widetilde{\mathbf{B}}^{(k)}=\left[\widetilde{\mathbf{b}}_{i j}^{(k)}\right]_{m \times n}$, where $\widetilde{\mathbf{b}}_{i j}^{(k)}=t_{a^{\sigma_{i j}}}^{(k)}, \sigma_{i j} \in$ $\{-f, \ldots, 0, \ldots, g\}$, and $i=1, \ldots, m, j=1, \ldots, n, k=$ $1, \ldots, d$. The 2-tuple linguistic representation of each linguistic term in the $d$ matrices is $\left(t_{a^{\sigma_{i j}}}^{(k)}, \gamma_{i j}^{(k)}\right)$, with $\gamma_{i j}^{(k)}=0$.

Without loss of generality we assume that in each matrix $\widetilde{\mathbf{B}}^{(k)}$, the first $\ell$-columns corresponding to the cost criteria (lower is better) and the remaining $n-\ell=s$ columns are beneficial criteria (higher is better).

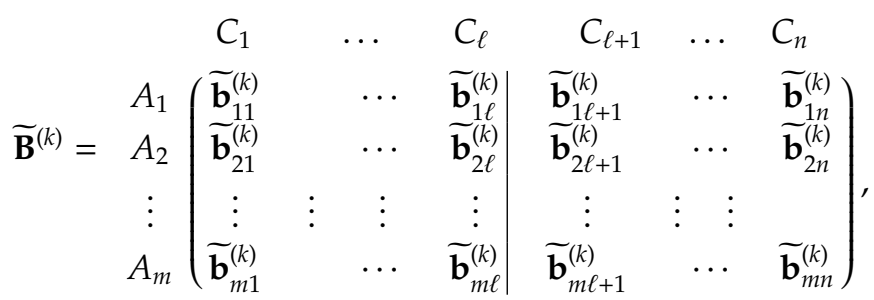

where $\widetilde{\mathbf{b}}_{i j}^{(k)}=\left(t_{a^{\sigma_{i j}}}^{(k)}, \gamma_{i j}^{(k)}=0\right), i=1, \ldots, m, j=1, \ldots, n$, $k=1, \ldots, d$.

The distinction in the criteria makes DEA a natural to implement in MCGDM problem. The cost criteria datavalues are considered as input data, while the beneficial criteria data values are the output data in the DEA model. The $m$ alternatives act as the $m$ DMUs. The DEA model computes the weights $w_{i j}^{(k)}$ of $k^{\text {th }}$ decision 
maker for the $i^{\text {th }}$ alternative on the $j^{\text {th }}$ criterion, $i=$ $1, \ldots, m, j=1, \ldots, n, k=1, \ldots, d$. We shall be using the index $p$ to denote the cost criteria and index $q$ for the beneficial criteria; $p=1, \ldots, \ell ; q=\ell+1, \ldots, \ell+s$.

We use the output oriented DEA model by Charnes et al. Charnes et al. (1978) for efficiency evaluation of a $\mathrm{DMU}_{o}$ as follows:

$$
\max \sum_{q=1}^{s} u_{o q}^{(k)} \Omega^{-1}\left(t_{a^{\sigma o q}}^{(k)}, 0\right)
$$

subject to

$$
\begin{aligned}
& \sum_{p=1}^{\ell} v_{o p}^{(k)} \Omega^{-1}\left(t_{a^{\sigma o p}}^{(k)}, 0\right)=1 \\
& \sum_{q=\ell+1}^{\ell+s} u_{i q}^{(k)} \Omega^{-1}\left(t_{a^{\sigma_{i q}}}^{(k)}, 0\right)-\sum_{p=1}^{\ell} v_{i p}^{(k)} \Omega^{-1}\left(t_{a^{\sigma_{i p}}}^{(k)}, 0\right) \leq 0, \\
& i=1, \ldots, m, \\
& u_{i q}^{(k)}, v_{i p}^{(k)} \geq 0, \\
& i=1, \ldots, m, p=1, \ldots, \ell, q=\ell+1, \ldots, \ell+s .
\end{aligned}
$$

$\operatorname{Model}(\mathrm{M} 1)$ is to be solved for each $\mathrm{DMU}_{o}, o=1, \ldots, m$, and for each decision maker $k=1, \ldots, d$. The optimal weights of $\ell$ inputs $v_{o p}^{(k)}$ and s outputs $u_{o q}^{(k)}$ are obtained for $D M U_{0}$. The optimal weight vector is $w_{i j}^{(k)}=$ $\left(u_{i p}^{(k)}, v_{i q}^{(k)}, p=1, \ldots, \ell\right), q=\ell+1, \ldots, \ell+s, i=1, \ldots, m, j=$ $1, \ldots, n=\ell+s, k=1, \ldots, d$. We normalize the optimal weight vector over the index $k$, and obtain the aggregated matrix $\mathbb{B}_{\text {agg }}=\left[\mathfrak{b}_{i j}\right]_{m \times n}$, with

$$
\begin{aligned}
\mathfrak{b}_{i j}=\Omega\left(\sum_{k=1}^{d} \frac{w_{i j}^{(k)}}{\sum_{k=1}^{d} w_{i j}^{(k)}} \Omega^{-1}\left(t_{a^{\sigma_{i j}}}^{(k)}, 0\right)\right), \\
\quad i=1, \ldots, m, j=1, \ldots, n .
\end{aligned}
$$

\section{Prospect Theory based Cross-Efficiency Evaluation}

Kahneman and Tversky (1979) put forward the prospect theory (PT) to model human behavior towards the preference structures or risk in the choices in an uncertain environment. The following three are the guiding principles behind their approach.

(i) Reference dependence. It means that concerning some reference point, one can distinguish outcomes into gains or losses. The prospect value curve domain gets segregated into the gain and loss domains. (ii) Loss aversion. Decision-makers have different risk appetites towards gains and losses. In a decision process, a decision maker is typically more sensitive to losses than to absolute commensurate gains. For this purpose, the prospect value curve is steeper in the loss domain than the gain domain.

(iii) Diminishing sensitivity. It portrays a risk-averse attitude when it comes to gains and a risk-seeking attitude when it means losses. The value curve is concave in the gain domain and convex in the loss domain. Both gains and losses experience a fall in their marginal values as their sizes grow large.

One of the most common prospect value functions $v(\Delta z)$ representing the subjective attitude of a decisionmaker is the power function, defined as follows:

$$
v(\Delta z)=\left\{\begin{array}{lc}
(\Delta z)^{\alpha} & \Delta z \geq 0, \\
-\theta(-\Delta z)^{\phi} & \Delta z<0 .
\end{array}\right.
$$

The departure of $z$ from the reference point $z_{0}$ is $\Delta z=z-$ $z_{0}$. It shows gains above $z_{0}$ and loses below $z_{0}$ through its non-negative and negative values. The parameters $\alpha \in(0,1)$ and $\phi \in(0,1)$ denote the bump degree in the gain domain and diminishing sensitivity in the loss domain of the power function, respectively. The greater values of $\alpha$ and $\phi$ indicate the greater risk appetite of the decision maker. The parameter $\theta>0$ is a lossaversion coefficient; $\theta>1$ indicates that the decisionmaker is more worrisome for losses than gains. Liu et al. (2014) suggested to take $\alpha=\phi=0.85$, and $\theta=4.1$. An experimental validation by Tversky and Kahneman (1992) advised to take $\alpha=0.89, \phi=0.92$, and $\theta=2.25$.

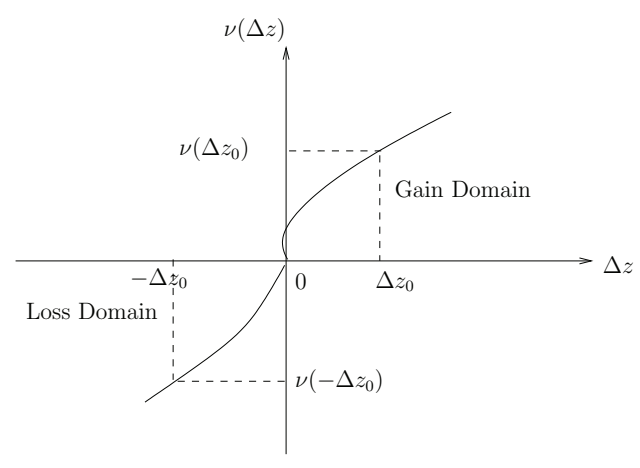

Fig. 1 Value function of prospect theory 
Tversky and Kahneman (1992) added a minor extensions to the prospect theory to present cumulative prospect theory (CPT) that employs cumulative rather than separable decision weights to the value function. The CPT has attracted attention in many diverse decision-making areas. For example, Harel et al. (2018) compared singleperiod utility of wealth functions with the value functions. Wang et al. (2020) introduced CPT into three way decision making theory and modify the weight functions to incorporate nonlinear transformation of the conditional probabilities. Liu and Li (2019) proposed an emergency group decision-making method with interval probability based on CPT. Zhao et al. (2021) presented a novel intuitionistic fuzzy CPT-TODIM method and implemented it on stock investment selection. Recently, Gao et al. (2021) investigated different weighting functions and power value functions in connecting the CPT with multi-attribute decision making for predicting travel behaviour. Gazioğlu and Çalışkan (2011) introduced a piecewise quadratic value function and showed it to be a superior approximation of Kahneman-Tversky value function than piecewise power and exponential value functions.

Using the worst and the best reference points in DEA, Liu et al. (2019) proposed a cross-efficiency model based on the prospect theory. The worst DMU uses maximum inputs to yield minimum outputs, while the best DMU does exactly the opposite by producing maximum outputs using the least inputs. The gain and loss of any DMU are measured relative to values above the worst DMU and below the best DMU.

In context of the MCGDM, the $i^{\text {th }}$ DMU input and output data values in $\mathbb{B}_{\text {agg }}$ matrix are respectively $\mathfrak{b}_{i p}, p=$ $1, \ldots, \ell$, and $b_{i q}, q=\ell+1, \ldots, \ell+s$.

Definition 4 Let the reference point be the worst DMU. The prospect gain value of $p^{\text {th }}$ input and $q^{\text {th }}$ output of $\mathrm{DMU}_{i}, i=1, \ldots, m$, are defined by

$$
\begin{aligned}
& \left(G_{i p}^{+}\right)_{I}=\left(\mathfrak{b}_{p}^{-}-\mathfrak{b}_{i p}\right)^{\alpha}, \quad p=1, \ldots, \ell, \\
& \left(G_{i q}^{+}\right)_{O}=\left(\mathfrak{b}_{i q}-\mathfrak{b}_{q}^{-}\right)^{\alpha}, \quad q=\ell+1, \ldots, \ell+s,
\end{aligned}
$$

where $\mathfrak{b}_{p}^{-}=\max _{1 \leq i \leq m}\left(\mathfrak{b}_{i p}\right)$ and $\mathfrak{b}_{q}^{-}=\min _{1 \leq i \leq m}\left(\mathfrak{b}_{i q}\right)$ are respectively the $p^{\text {th }}$ input and $q^{\text {th }}$ output of the worst DMU.
Definition 5 Let the reference point be the best DMU. The prospect loss values of $p^{\text {th }}$ input and $q^{\text {th }}$ output of $\mathrm{DMU}_{i}, i=1, \ldots, m$, are defined by

$$
\begin{aligned}
& \left(L_{i p}^{-}\right)_{I}=-\theta\left(\mathfrak{b}_{i p}-\mathfrak{b}_{p}^{+}\right)^{\phi}, \quad p=1, \ldots, \ell, \\
& \left(L_{i q}^{-}\right)_{O}=-\theta\left(\mathfrak{b}_{q}^{+}-\mathfrak{b}_{i q}\right)^{\phi}, \quad q=\ell+1, \ldots, \ell+s,
\end{aligned}
$$

where $\mathfrak{b}_{p}^{+}=\min _{1 \leq i \leq m}\left(\mathfrak{b}_{i p}\right)$ and $\mathfrak{b}_{q}^{+}=\max _{1 \leq i \leq m}\left(\mathfrak{b}_{i q}\right)$ are respectively the $p^{\text {th }}$ input and $q^{\text {th }}$ output of the best DMU.

The decision maker always prefer to allocate weights to inputs and outputs so to maximize gain and minimize loss for $\mathrm{DMU}_{i}, i=1, \ldots, m$. To accomplish this task, Liu et al. (2019) proposed the following cross-efficiency model for $\mathrm{DMU}_{i}$ :

$$
\begin{aligned}
\max & \eta\left(\sum_{q=\ell+1}^{\ell+s} \mathbb{u}_{i q}\left(G_{i q}^{+}\right)_{O}+\sum_{p=1}^{\ell} \mathbb{w}_{i p}\left(G_{i p}^{+}\right)_{I}\right) \\
& -(1-\eta)\left(\sum_{q=\ell+1}^{\ell+s} \mathbb{w}_{i q}\left(L_{i q}^{-}\right)_{O}+\sum_{p=1}^{\ell} \mathbb{w}_{i p}\left(L_{i p}^{-}\right)_{I}\right)
\end{aligned}
$$

subject to

$$
\begin{aligned}
& \sum_{p=1}^{l} \mathbb{w}_{i p} \mathfrak{b}_{i p}=1, \\
& \sum_{q=\ell+1}^{\ell+s} \mathbb{u}_{i q} \mathfrak{b}_{i q}=1, \\
& \sum_{q=\ell+1}^{\ell+s} \mathbb{u}_{i q} \mathfrak{b}_{i q}-\sum_{p=1}^{l} \mathbb{v}_{i p} \mathfrak{b}_{i p} \leq 0, \quad i=1, \ldots, m, \\
& \mathbb{u}_{i q}, \mathbb{w}_{i p} \geq 0, \quad p=1, \ldots, \ell, q=\ell+1, \ldots, \ell+s .
\end{aligned}
$$

Here, $\eta \in[0,1]$ depicts the trade-off parameter between the prospect gain and prospect loss for the decision maker.

\section{Proposed Methodology}

Liu et al. (2019) defined cross-efficiency of DMUs based on prospect theory. Wu et al. (2012) proposed to use Shannon entropy for aggregating efficiencies in crossefficiency matrix. The entropy model generated weights for aggregating and explaining the final cross-efficiency values of DMUs. Song and Liu (2018) noticed that the weights produced by $\mathrm{Wu}$ et al. (2012) approach are inconsistent with acceptable views. They suggested an 
improvement in the method and introduced a variation coefficient approach for DEA cross-efficiency aggregation based on Shannon entropy.

Taking motivation from the cited works, in this section, we put forward a procedure to solve MCGDM problem with entries coming from the unbalanced 2-tuple linguistic term set.

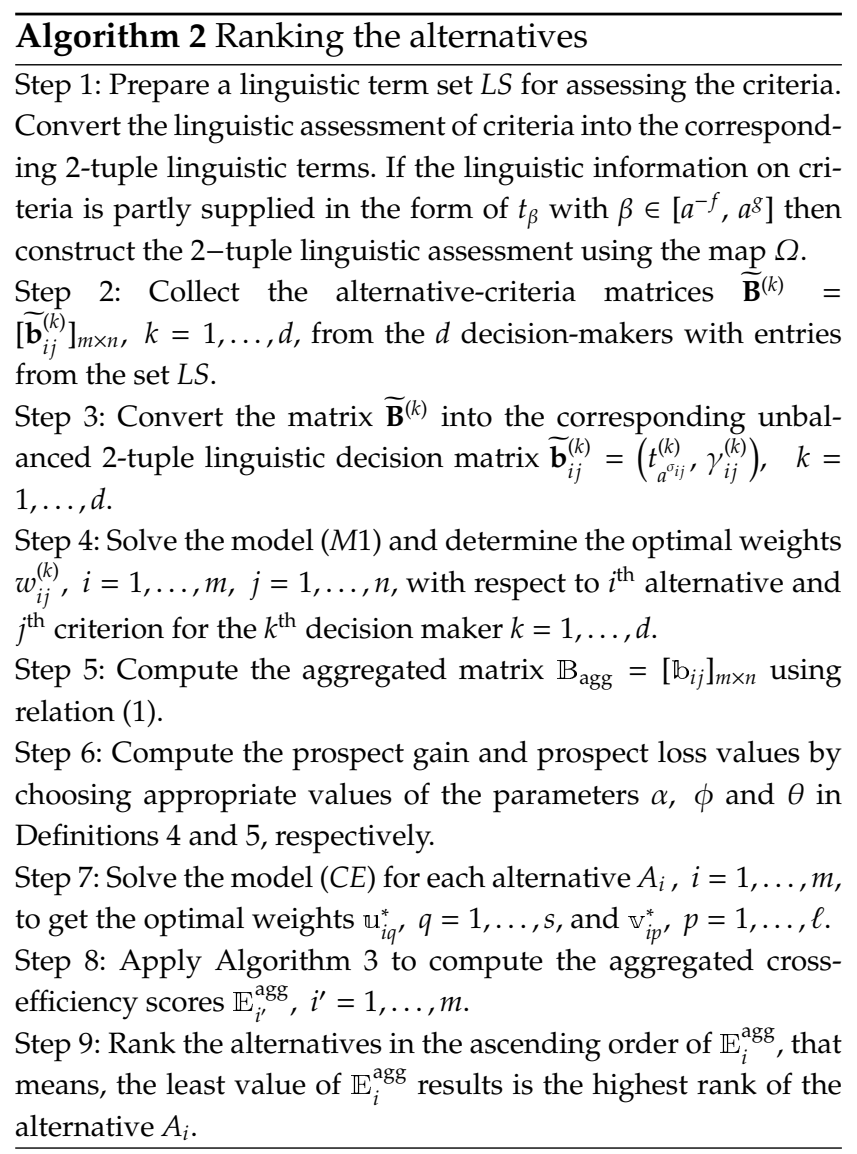

Algorithm 3 Aggregated cross-efficiency scores

Step 1: Compute the cross-efficiency $\mathbb{E}_{i i^{\prime}}$ of $\mathrm{DMU}_{i^{\prime}}$ using the optimal weights $\mathbb{d}_{i q}^{*}$ and $\mathbb{v}_{i p}^{*}$ by $(C E)$ model for $\mathrm{DMU}_{i}$.

$$
\mathbb{E}_{i i^{\prime}}=\sum_{q=1}^{s} \mathbb{u}_{i q}^{*} b_{i^{\prime} q} / \sum_{p=1}^{\ell} \mathbb{v}_{i p}^{*} b_{i^{\prime} p}, \quad i, i^{\prime}=1, \ldots, m .
$$

Step 2: Compute Shannon's entropy of $E_{i i^{\prime}}$ defined by

$e_{i i^{\prime}}=-\left(\mathbb{E}_{i i^{\prime}} / \sum_{i^{\prime}=1}^{m} \mathbb{E}_{i i^{\prime}}\right) \ln \left(\mathbb{E}_{i i^{\prime}} / \sum_{i^{\prime}=1}^{m} \mathbb{E}_{i i^{\prime}}\right), \quad i, i^{\prime}=1, \ldots, m$.

Step 3: Compute the mean and standard deviation of entropy for $\mathrm{DMU}_{i}, i=1, \ldots, m$, as

$$
\bar{e}_{i}=\frac{1}{m} \sum_{i^{\prime}=1}^{m} e_{i i^{\prime}}, \quad \pi_{i}=\sqrt{\frac{1}{m} \sum_{i^{\prime}=1}^{m}\left(e_{i i^{\prime}}-\bar{e}_{i}\right)^{2}} .
$$

Step 4: Compute the variation coefficients $\delta_{i}$ and normalize it to find the cross-efficiency weights of alternative $A_{i}$ as $\xi_{i}$.

$$
\delta_{i}=\frac{\pi_{i}}{\bar{e}_{i}}, \quad \xi_{i}=\frac{\delta_{i}}{\sum_{i=1}^{m} \delta_{i}}, \quad i=1, \ldots, m .
$$

Step 5: The aggregated cross-efficiency of $\mathrm{DMU}_{i^{\prime}}$ is defined by $\mathbb{E}_{i^{\prime}}^{\mathrm{agg}}=\sum_{i=1}^{m} \xi_{i} \mathbb{E}_{i i^{\prime}}, \quad i^{\prime}=1, \ldots, m$.

\section{Illustration}

We present an example to showcase the proposed methodology. The example is set in the backdrop of medical devices selection. Medical devices are expensive and incur a high maintenance cost. Selection of the best medical device from available options is a crucial issue for a hospital. Suppose a hospital $\mathbb{H}$ wants to improve its technological level and to achieve the level it is required to purchase a set of sensor devices by the hospital. Medical experts have shortlisted eight sensor devices $\left\{A_{i}, i=1, \ldots, 8\right\}$. Seven criteria are identified for assessment: maintenance support difficulty $\left(C_{1}\right)$, purchase cost $\left(C_{2}\right)$, degree of environmental interference $\left(C_{3}\right)$, stability $\left(C_{4}\right)$, sensitivity $\left(C_{5}\right)$, linear range $\left(C_{6}\right)$, and the degree of intelligence $\left(C_{7}\right)$. Among these, $C_{1}, C_{2}$, and $C_{3}$ are cost criteria thus considered as inputs while $C_{4}, C_{5}, C_{6}$, and $C_{7}$ are beneficial criteria taken as outputs in DEA model (M1). Four decisionmakers $D_{k}, k=1, \ldots, 4$, evaluate eight alternatives on seven criteria from the linguistic term set with the following semantic representation: 
$L S=\left\{t_{2^{(-4)}}:\right.$ very disappointing $(\mathbb{V D})$,

$t_{2^{(-3)}}$ : disappointing $(\mathbb{D}), t_{2^{(-2)}}$ : unsatisfactory $(\mathbb{U})$,

$t_{2^{(-1)}}$ : partly unsatisfactory $(\mathbb{P} \mathbb{U})$,

$t_{2^{(0)}}$ : just right $(\mathbb{J R}), t_{2^{(1)}}:$ slightly satisfactory $(\mathbb{S} \mathbb{S})$,

$t_{2^{(2)}}$ : satisfactory $(\mathbb{S}), t_{2^{(3)}}$ : more than satisfactory $(\mathbb{M} \mathbb{S})$,

$t_{2^{(3)}}:$ very satisfactory $\left.(\mathbb{V} \mathbb{S})\right\}$.

Table 1 exhibits the alternative-criteria assessment matrices with entries provided by the decision-makers from the set $L S$.

Table 1 Alternative-criteria assessment by four decision-makers (read in the order of the tuple from left to right)

\begin{tabular}{|c|c|c|c|c|c|c|c|}
\hline & $C_{1}$ & $C_{2}$ & $C_{3}$ & $C_{4}$ & $C_{5}$ & $C_{6}$ & $C_{7}$ \\
\hline \multirow[t]{2}{*}{$A_{1}$} & $\mathbb{S} \mathbb{S}, \mathbb{P} \mathbb{U}$ & $\mathbb{S}, \mathbb{P} \mathbb{U}$ & $\mathbb{J} \mathbb{R}, \mathbb{J} \mathbb{R}$ & $\mathbb{S}, \mathbb{P} \mathbb{U}$ & $\mathbb{S} \mathbb{S}, \mathbb{P} \mathbb{U}$ & $\mathbb{D} \mathbb{R}, \mathbb{D} \mathbb{R}$ & $\mathbb{S}, \mathbb{S}$ \\
\hline & $\mathbb{J} \mathbb{R}, \mathbb{U}$ & $\mathbb{S}, \mathbb{P} \mathbb{U}$ & $\mathbb{U}, \mathbb{S} \mathbb{S}$ & $\mathbb{P} \mathbb{U}, \mathbb{U}$ & $\mathbb{U}, \mathbb{S}$ & $\mathbb{P} \mathbb{U}, \mathbb{D}$ & $\mathbb{U}, \mathbb{P} \mathbb{U}$ \\
\hline \multirow[t]{2}{*}{$A_{2}$} & $\mathbb{P} \mathbb{U}, \mathbb{D}$ & $\mathbb{S} \mathbb{S}, \mathbb{P} \mathbb{U}$ & $\mathbb{S}, \mathbb{P} \mathbb{U}$ & $\mathbb{S}, \mathbb{S} \mathbb{S}$ & $\mathbb{S}, \mathbb{D} \mathbb{R}$ & $\mathbb{J} \mathbb{R}, \mathbb{D}$ & $\mathbb{S} \mathbb{S}, \mathbb{V D}$ \\
\hline & $\mathbb{D}, \mathbb{D} \mathbb{R}$ & $\mathbb{S} \mathbb{S}, \mathbb{D} \mathbb{R}$ & $\mathbb{D}, \mathbb{U}$ & $\mathbb{D}, \mathbb{J} \mathbb{R}$ & $\mathbb{D}, \mathbb{P} \mathbb{U}$ & $\mathbb{D}, \mathbb{J} \mathbb{R}$ & $\mathbb{D}, \mathbb{D}$ \\
\hline \multirow[t]{2}{*}{$A_{3}$} & $\mathbb{M} \mathbb{S}, \mathbb{V} \Phi$ & $\mathbb{M} \mathbb{S}, \mathbb{V} \mathbb{S}$ & $\mathbb{S}, \mathbb{S} \mathbb{S}$ & $\mathbb{S}, \mathbb{S}$ & $\mathbb{V} \mathbb{S}, \mathbb{M} \Phi$ & $\mathbb{M} \mathbb{S}, \mathbb{M} \mathbb{S}$ & $\mathbb{S}, \mathbb{V} \mathbb{S}$ \\
\hline & $\mathbb{P} \mathbb{U}, \mathbb{I} \mathbb{R}$ & $\mathbb{S}, \mathbb{M} \mathbb{S}$ & $\mathbb{S}, \mathbb{S}$ & $\mathbb{S}, \mathbb{M} \mathbb{S}$ & $\mathbb{I} \mathbb{R}, \mathbb{S} \mathbb{S}$ & $\mathbb{V} \mathbb{S}, \mathbb{S}$ & $\mathbb{P} \mathbb{U}, \mathbb{S} \mathbb{S}$ \\
\hline \multirow[t]{2}{*}{$A_{4}$} & $\mathbb{S}, \mathbb{P} \mathbb{U}$ & $\mathbb{M} \mathbb{S}, \mathbb{U}$ & $\mathbb{S} \mathbb{S}, \mathbb{S}$ & $\mathbb{S}, \mathbb{D}$ & $\mathbb{S}, \mathbb{M} \mathbb{S}$ & $\mathbb{M} \mathbb{S}, \mathbb{S}$ & $\mathbb{S} \mathbb{S}, \mathbb{V} \mathbb{S}$ \\
\hline & $\mathbb{V} \mathbb{D}, \mathbb{S} \mathbb{S}$ & $\mathbb{D}, \mathbb{I} \mathbb{R}$ & $\mathbb{J} \mathbb{R}, \mathbb{V} \mathbb{D}$ & $\mathbb{P} \mathbb{U}, \mathbb{D}$ & $\mathbb{U}, \mathbb{S}$ & $\mathbb{S} \mathbb{S}, \mathbb{P} \mathbb{U}$ & $\mathbb{S} \mathbb{S}, \mathbb{Z} \mathbb{R}$ \\
\hline \multirow[t]{2}{*}{$A_{5}$} & $\mathbb{M} \mathbb{S}, \mathbb{M} \mathbb{S}$ & $\mathbb{S}, \mathbb{J} \mathbb{R}$ & $\mathbb{M} \mathbb{S}, \mathbb{J} \mathbb{R}$ & $\mathbb{S} \mathbb{S}, \mathbb{S} \mathbb{S}$ & $\mathbb{J} \mathbb{R}, \mathbb{P} \mathbb{U}$ & $\mathbb{U}, \mathbb{P} \mathbb{U}$ & $\mathbb{V} \mathbb{S}, \mathbb{M} \mathbb{S}$ \\
\hline & $\mathbb{J} \mathbb{R}, \mathbb{P} \mathbb{U}$ & $\mathbb{U}, \mathbb{S} \mathbb{S}$ & $\mathbb{P} \mathbb{U}, \mathbb{I} \mathbb{R}$ & $\mathbb{P} \mathbb{U}, \mathbb{J} \mathbb{R}$ & $\mathbb{S}, \mathbb{S}$ & $\mathbb{D}, \mathbb{S}$ & $\mathbb{S}, \mathbb{J} \mathbb{R}$ \\
\hline \multirow[t]{2}{*}{$A_{6}$} & $\mathbb{J} \mathbb{R}, \mathbb{S}$ & $\mathbb{S}, \mathbb{S} \mathbb{S}$ & $\mathbb{V} \mathbb{S}, \mathbb{M} \mathbb{S}$ & $\mathbb{S}, \mathbb{M} \mathbb{S}$ & $\mathbb{S} \mathbb{S}, \mathbb{M} \mathbb{S}$ & $\mathbb{S}, \mathbb{S} \mathbb{S}$ & $\mathbb{P} \mathbb{U}, \mathbb{J} \mathbb{R}$, \\
\hline & $\mathbb{S} \mathbb{S}, \mathbb{P} \mathbb{U}$ & $\mathbb{S}, \mathbb{M} \mathbb{S}$ & $\mathbb{S}, \mathbb{S} \mathbb{S}$ & $\mathbb{P} \mathbb{U}, \mathbb{U}$ & $\mathbb{V} \mathbb{S}, \mathbb{S}$ & $\mathbb{J} \mathbb{R}, \mathbb{V} \mathbb{S}$ & $\mathbb{S}, \mathbb{D}$ \\
\hline \multirow[t]{2}{*}{$A_{7}$} & $\mathbb{S} \mathbb{S}, \mathbb{S} \mathbb{S}$ & $\mathbb{S} \mathbb{S}, \mathbb{S}$ & $\mathbb{S}, \mathbb{S} \mathbb{S}$ & $\mathbb{S}, \mathbb{S} \mathbb{S}$ & $\mathbb{S} \mathbb{S}, \mathbb{J} \mathbb{R}$ & $\mathbb{S}, \mathbb{M} \mathbb{S}$ & $\mathbb{S} \mathbb{S}, \mathbb{D} \mathbb{R}$ \\
\hline & $\mathbb{S}, \mathbb{S} \mathbb{S}$ & $\mathbb{J} \mathbb{R}, \mathbb{V} \mathbb{S}$ & $\mathbb{J} \mathbb{R}, \mathbb{M} \mathbb{S}$ & $\mathbb{S}, \mathbb{D}$ & $\mathbb{S} \mathbb{S}, \mathbb{D} \mathbb{R}$ & $\mathbb{M} \mathbb{S}, \mathbb{M} \mathbb{S}$ & $\mathbb{D} \mathbb{R}, \mathbb{S}$ \\
\hline \multirow[t]{2}{*}{$A_{8}$} & $\mathbb{P} \mathbb{U}, \mathbb{J} \mathbb{R}$ & $\mathbb{S} \mathbb{S}, \mathbb{S} \mathbb{S}$ & $\mathbb{S}, \mathbb{U}$ & $\mathbb{J} \mathbb{R}, \mathbb{Z} \mathbb{R}$ & $\mathbb{V} \mathbb{S}, \mathbb{P} \mathbb{U}$ & $\mathbb{S}, \mathbb{U}$ & $\mathbb{U}, \mathbb{P} \mathbb{U}$ \\
\hline & $\mathbb{J} \mathbb{R}, \mathbb{D} \mathbb{R}$ & $\mathbb{J} \mathbb{R}, \mathbb{U}$ & $\mathbb{P} \mathbb{U}, \mathbb{P} \mathbb{U}$ & $\mathbb{J} \mathbb{R}, \mathbb{U}$ & $\mathbb{S} \mathbb{S}, \mathbb{D} \mathbb{R}$ & $\mathbb{D}, \mathbb{S} \mathbb{S}$ & $\mathbb{D} \mathbb{R}, \mathbb{D} \mathbb{R}$ \\
\hline
\end{tabular}

Table 2 reports the optimal weights for alternativecriteria of the decision-makers on solving model (M1). The aggregated matrix $\mathbb{B}_{\text {agg }}$ obtained is as follows:
Table 2 Optimal weights by model (M1) on alternative-criteria matrices of the decision-makers.

\begin{tabular}{|c|c|c|c|c|c|c|c|c|}
\hline $\begin{array}{c}\text { Matr- } \\
\text { ices }\end{array}$ & $\begin{array}{l}\text { Alter- } \\
\text { natives }\end{array}$ & $C_{1}$ & $C_{2}$ & $C_{3}$ & $C_{4}$ & $C_{5}$ & $C_{6}$ & $C_{7}$ \\
\hline \multirow{8}{*}{$\widetilde{\mathbf{B}}^{(1)}$} & $A_{1}$ & 0 & 0.2 & 0.182 & 0.072 & 0.031 & 0.13 & 0.13 \\
\hline & $A_{2}$ & 0 & 0.19 & 0.16 & 0.2 & 0.05 & 0.03 & 0 \\
\hline & $A_{3}$ & 0 & 0.088 & 0.073 & 0 & 0.005 & 0.093 & 0.04 \\
\hline & $A_{4}$ & 0 & 0.105 & 0.0714 & 0 & 0 & 0.125 & 0 \\
\hline & $A_{5}$ & 0 & 0.135 & 0.057 & 0.1 & 0 & 0 & 0.05 \\
\hline & $A_{6}$ & 0.2 & 0.2 & 0 & 0.1 & 0 & 0.1 & 0 \\
\hline & $A_{7}$ & 0 & 0.214 & 0.14 & 0 & 0 & 0.25 & 0 \\
\hline & $A_{8}$ & 0 & 0 & 0.25 & 0 & 0.063 & 0 & 0 \\
\hline \multirow{8}{*}{$\widetilde{\mathbf{B}}^{(2)}$} & $A_{1}$ & 0.52 & 0 & 0.74 & 0.2 & 0 & 0.15 & 0.16 \\
\hline & $A_{2}$ & 0 & 0 & 2 & 0 & 1 & 0 & 0 \\
\hline & $A_{3}$ & 0 & 0 & 0.125 & 0.005 & 0.035 & 0.008 & 0.011 \\
\hline & $A_{4}$ & 0 & 0 & 0.25 & 0 & 0.125 & 0 & 0 \\
\hline & $A_{5}$ & 0.325 & 0 & 0.36 & 0.11 & 0 & 0 & 0.0984 \\
\hline & $A_{6}$ & 0 & 0.45 & 0.012 & 0.11 & 0.018 & 0 & 0 \\
\hline & $A_{7}$ & 0 & 0.23 & 0.094 & 0.073 & 0 & 0.1 & 0 \\
\hline & $A_{8}$ & 0 & 0.09 & 3.25 & 0.32 & 0.99 & 0 & 0.26 \\
\hline \multirow{8}{*}{$\widetilde{\mathbf{B}}^{(3)}$} & $A_{1}$ & 0.67 & 0 & 1.33 & 0.88 & 0.22 & 0 & 0 \\
\hline & $A_{2}$ & 3.15 & 0.09 & 3.3 & 3.62 & 0.52 & 0 & 0.23 \\
\hline & $A_{3}$ & 0.09 & 0 & 0.24 & 0.011 & 0.05 & 0.06 & 0 \\
\hline & $A_{4}$ & 0.253 & 0 & 0.98 & 0 & 0 & 0.25 & 0 \\
\hline & $A_{5}$ & 0.78 & 0 & 0.43 & 0.49 & 0.19 & 0 & 0 \\
\hline & $A_{6}$ & 0.5 & 0 & 0 & 0.04 & 0.04 & 0 & 0.08 \\
\hline & $A_{7}$ & 0.2 & 0.06 & 0.21 & 0.23 & 0.04 & 0 & 0 \\
\hline & $A_{8}$ & 0.68 & 0.013 & 0.62 & 0.67 & 0.16 & 0 & 0 \\
\hline \multirow{8}{*}{$\widetilde{\mathbf{B}}^{(4)}$} & $A_{1}$ & 0.39 & 0.2 & 0.4 & 0 & 0.25 & 0 & 0 \\
\hline & $A_{2}$ & 0.61 & 0 & 1.55 & 0.71 & 0 & 0.29 & 0 \\
\hline & $A_{3}$ & 0.023 & 0.094 & 0.055 & 0.113 & 0.032 & 0 & 0.013 \\
\hline & $A_{4}$ & 0.356 & 0.27 & 0.28 & 0 & 0.144 & 0 & 0.42 \\
\hline & $A_{5}$ & 0.59 & 0.35 & 0 & 0.27 & 0 & 0.06 & 0.5 \\
\hline & $A_{6}$ & 0.29 & 0.054 & 0 & 0 & 0 & 0.063 & 0 \\
\hline & $A_{7}$ & 0.5 & 0 & 0 & 0 & 0 & 0 & 0.25 \\
\hline & $A_{8}$ & 0.9 & 0.24 & 0.08 & 0 & 0 & 0 & 1 \\
\hline
\end{tabular}




$$
\left(\begin{array}{ccccccc}
0.651 & 2.237 & 0.775 & 0.719 & 2.23 & 1 & 4 \\
0.267 & 2 & 0.346 & 0.533 & 0.802 & 1 & 0.125 \\
0.603 & 4.377 & 5.019 & 7.504 & 3.909 & 10.854 & 5.631 \\
1.196 & 2.96 & 1.350 & 0 & 5.859 & 4 & 1 \\
1.017 & 2.556 & 1.222 & 0.965 & 4 & 4 & 3.23 \\
1.363 & 3.025 & 8 & 5.136 & 13.559 & 8.615 & 4 \\
2.563 & 2.789 & 1.955 & 3.52 & 2 & 5.143 & 4 \\
1 & 0.756 & 2.536 & 1 & 7.433 & 0 & 0.896
\end{array}\right)
$$

We take $\eta=0.5, \alpha=0.89, \phi=0.92$ and $\theta=2.25$, and solve $(C E)$ model for each of the alternatives. The parameters values are set following Kahneman and Tversky (1979), while in the absence of any information on the trade-off between loss and gain, we set $\eta=0.5$. Table 3 exhibits the optimal weights from (CE) model and Table 4 provides the cross-efficiency values $\mathbb{E}_{i i^{\prime}}$, $i, i^{\prime}=1, \ldots, 8$.

Table 3 Optimal solution of model (CE) for each alternatives.

\begin{tabular}{cccccccc}
\hline & $C_{1}$ & $C_{2}$ & $C_{3}$ & $C_{4}$ & $C_{5}$ & $C_{6}$ & $C_{7}$ \\
\hline$A_{1}$ & 1.403 & 0 & 0.112 & 0 & 0 & 0 & 0.25 \\
\hline$A_{2}$ & 0.94 & 0 & 2.166 & 0.341 & 0 & 0.818 & 0 \\
\hline$A_{3}$ & 1.659 & 0 & 0 & 0 & 0 & 0.092 & 0 \\
\hline$A_{4}$ & 0.49 & 0 & 0.307 & 0 & 0.171 & 0 & 0 \\
\hline$A_{5}$ & 0.722 & 0 & 0.218 & & 0.1473 & 0.061 & 0.052 \\
\hline$A_{6}$ & 0.734 & 0 & 0 & 0 & 0.074 & 0 & 0 \\
\hline$A_{7}$ & 0 & 0.003 & 0.507 & 0.0849 & 0 & 0.136 & 0 \\
\hline$A_{8}$ & 0 & 1.322 & 0 & 0 & 0.135 & 0 & 0 \\
\hline
\end{tabular}

Table 4 The cross-efficiency $\mathbb{E}_{i i^{\prime}}, i, i^{\prime}=1, \ldots, 8$, for the alternatives.

\begin{tabular}{ccccccccc}
\hline & $A_{1}$ & $A_{2}$ & $A_{3}$ & $A_{4}$ & $A_{5}$ & $A_{6}$ & $A_{7}$ & $A_{8}$ \\
\hline$A_{1}$ & 1 & 0.076 & 1 & 0.137 & 0.516 & 0.356 & 0.262 & 0.133 \\
\hline$A_{2}$ & 0.464 & 1 & 1 & 0.81 & 1 & 0.473 & 0.814 & 0.053 \\
\hline$A_{3}$ & 0.085 & 0.208 & 1 & 0.186 & 0.218 & 0.351 & 0.111 & 0 \\
\hline$A_{4}$ & 0.684 & 0.578 & 0.363 & 1 & 0.782 & 0.741 & 0.184 & 1 \\
\hline$A_{5}$ & 0.936 & 0.691 & 0.999 & 0.999 & 1 & 1 & 0.358 & 0.895 \\
\hline$A_{6}$ & 0.344 & 0.302 & 0.652 & 0.493 & 0.395 & 1 & 0.079 & 0.747 \\
\hline$A_{7}$ & 0.493 & 0.998 & 0.827 & 0.786 & 0.999 & 0.396 & 1 & 0.066 \\
\hline$A_{8}$ & 0.101 & 0.041 & 0.091 & 0.201 & 0.16 & 0.456 & 0.073 & 1 \\
\hline
\end{tabular}

We next compute the entropy values of the cross efficiencies, and list the same in Table 5.

Table 5 Entropy $e_{i i^{\prime}}, i, i^{\prime}=1, \ldots, 8$, of cross-efficiency values.

\begin{tabular}{ccccccccc}
\hline & $A_{1}$ & $A_{2}$ & $A_{3}$ & $A_{4}$ & $A_{5}$ & $A_{6}$ & $A_{7}$ & $A_{8}$ \\
\hline$A_{1}$ & 0.358 & 0.083 & 0.358 & 0.127 & 0.283 & 0.233 & 0.195 & 0.125 \\
\hline$A_{2}$ & 0.206 & 0.307 & 0.307 & 0.279 & 0.307 & 0.208 & 0.280 & 0.044 \\
\hline$A_{3}$ & 0.128 & 0.225 & 0.357 & 0.211 & 0.232 & 0.295 & 0.153 & 0 \\
\hline$A_{4}$ & 0.263 & 0.241 & 0.183 & 0.314 & 0.282 & 0.274 & 0.116 & 0.314 \\
\hline$A_{5}$ & 0.271 & 0.231 & 0.280 & 0.280 & 0.280 & 0.280 & 0.154 & 0.265 \\
\hline$A_{6}$ & 0.211 & 0.195 & 0.295 & 0.258 & 0.228 & 0.346 & 0.077 & 0.313 \\
\hline$A_{7}$ & 0.215 & 0.308 & 0.283 & 0.276 & 0.308 & 0.188 & 0.308 & 0.053 \\
\hline$A_{8}$ & 0.145 & 0.076 & 0.135 & 0.223 & 0.194 & 0.330 & 0.116 & 0.355 \\
\hline
\end{tabular}

Table 6 presents the cross-efficiency weights $\xi, i=$ $1, \ldots, 8$, of alternatives, the aggregated cross -efficiency scores, and ranks of alternatives.

Table 6 Aggregated cross-efficiency and ranking of alternatives.

\begin{tabular}{cccc}
\hline & $\xi_{i}$ & $\mathbb{E}_{i}^{\mathrm{agg}}$ & Rank \\
\hline$A_{1}$ & 0.157 & 0.457 & 5 \\
$A_{2}$ & 0.121 & 0.421 & 7 \\
$A_{3}$ & 0.1767 & 0.732 & 1 \\
$A_{4}$ & 0.089 & 0.481 & 4 \\
$A_{5}$ & 0.056 & 0.558 & 2 \\
$A_{6}$ & 0.114 & 0.535 & 3 \\
$A_{7}$ & 0.119 & 0.337 & 8 \\
$A_{8}$ & 0.166 & 0.426 & 6 \\
\hline
\end{tabular}

Keeping the parameters $\alpha=0.89, \phi=0.92$ and $\theta=$ 2.25 , at the same levels, we vary the trade-off parameters $\eta \in(0,1)$, to see the change it brought in the ranks of the alternatives. The results are summarized in Table 7. 
Table 7 Ranks of alternatives for different values of $\eta$ in $(0,1)$.

\begin{tabular}{c|ccccccccc}
\hline $\begin{array}{c}\eta \rightarrow \\
\text { Alternatives } \downarrow\end{array}$ & 0.1 & 0.2 & 0.3 & 0.4 & 0.5 & 0.6 & 0.7 & 0.8 & 0.9 \\
\hline$A_{1}$ & 1 & 3 & 1 & 3 & 5 & 2 & 3 & 3 & 2 \\
$A_{2}$ & 8 & 8 & 8 & 8 & 7 & 8 & 8 & 8 & 8 \\
$A_{3}$ & 2 & 1 & 2 & 1 & 1 & 1 & 1 & 1 & 1 \\
$A_{4}$ & 7 & 7 & 7 & 7 & 4 & 7 & 7 & 7 & 7 \\
$A_{5}$ & 5 & 6 & 5 & 5 & 2 & 4 & 5 & 5 & 4 \\
$A_{6}$ & 3 & 2 & 3 & 2 & 3 & 3 & 2 & 2 & 3 \\
$A_{7}$ & 6 & 5 & 6 & 6 & 8 & 6 & 6 & 6 & 6 \\
$A_{8}$ & 4 & 4 & 4 & 4 & 6 & 5 & 4 & 4 & 5
\end{tabular}

The different values of the parameter $\eta$ capturing different perspectives towards gain and loss yield different rankings of alternatives that cannot be ignored. We apply Kendall's coefficient of concordance test to test the statistical significance of rank change for the pessimistic and optimistic situations. Kendall's coefficient of concordance assesses an agreement between quantitative variables used to evaluate a collection of a finite number of items of interest. The test statistics $W$ is calculated as follows:

$$
W=\frac{12 \sum_{i=1}^{m}\left(R_{i}-\bar{R}\right)^{2}}{\tau^{2}\left(m^{3}-m\right)},
$$

where $R_{i}$ is the sum of ranks for the $i^{\text {th }}$ object judged by $\tau$ assessing variables, $\bar{R}$ is the mean of all $R_{i}$ values, $\tau$ is the number of judgments on $m$ objects. The random variable $\chi^{2}=\tau(m-1) W$ is asymptotically chi-square distributed with $m-1$ degrees of freedom.

We first consider the pessimistic case when

$\eta=0.1,0.2,0.3,0.4,0.5$, so, $\tau=5$ (judges) and $m=8$ alternatives $\left(A_{1}, \ldots, A_{8}\right)$. The null hypothesis and the alternative hypothesis are set as follows:

$H_{\text {pess }}^{0}$ : there is no concordance in rankings of alternatives in pessimistic case;

$H_{\text {pess }}^{1}$ : there is a concordance in rankings of alternatives in pessimistic case.

The test statistics $W=0.81524$, and $\chi^{2}=28.5334$ with $p$-value equals 0.000176 . The null hypothesis gets rejected. On the similar lines, in the optimistic case when $\eta=0.5,0.6,0.7,0.8,0.9$, the test statistics $W=0.87$, and $\chi^{2}=30.47$ with the $p$-value equals 0.000078 . The null hypothesis of no concordance in rankings of alternatives in optimistic case also gets rejected.

\section{Conclusions}

The highlighting contributions of our paper are fourfolds. Firstly, we have used the 2-tuple unbalanced linguistic term set that provides a broader and more realistic domain set for the decision-makers in supplying the alternative-criteria judgment matrices. Unlike several other studies on multi-criteria decision-making demanding precise numerical quantification, the linguistic terms embrace decision-makers' multi-granularity and cognitive process in comprehending the available alternatives on different criteria.

Secondly, we present the DEA optimization model to compute the weights of the decision-makers on each alternative-criteria pair in the group decision-making. The DEA model incorporates complete information from the input criteria (or cost criteria) and output criteria (or beneficial criteria) from the decision matrices to designate weights that enable the computation of one aggregated decision matrix.

Thirdly, taking inspiration from the well-known TOPSIS method for MCDM, we determine the positive ideal (the best) and negative ideal (the worst) alternatives and use the prospect theory to determine the alternatives' gain and loss values concerning the two ideal options. The prospect theory value function allows for segregating the gain and loss domains based on the risk perspective, where guaranteed small gains are preferred over uncertain large gains and large uncertain losses get preferred over certain small losses.

Finally, we put forward a cross-efficiency DEA optimization model and aggregation of cross-efficiency scores to rank the alternatives.

The proposed methodology offers a comprehensive package that includes ease for decision-makers in providing their judgment on alternative-criteria in the quantitative form rather than numeric; computing the weights of decision-makers on alternative-criteria rather than knowing them prior; imparting more weights to the distance measure of alternatives from the ideal options on the cost criteria than to the benefit ones by utilizing the prospect theory value function; finally including cross-efficiency to rank the alternatives. 
We present a numerical example to illustrate the proposed methodology. The rankings obtained on varying the parameter in the cross-efficiency model get statistically tested for concordance or agreement. The null hypothesis of no concordance is rejected at a very high confidence level, thereby showing the efficacy and robustness of the proposed methodology in ranking the alternatives both in the pessimistic and optimistic situations.

For future works, a promising research avenue could be to use personalized individual semantics to represent the distinct understanding of each linguistic term by individual decision-maker. We can also explore using the utility function of cumulative prospect theory in MCGDM. In addition, we can employ different crossefficiency data envelopment analysis models, including satisfaction and consensus information for ranking.

\section{Ethical approval}

The research does not contain any studies performed with human participants or animals by any of the authors.

\section{Informed consent}

The research does not involve any human participants and animals.

\section{Funding details}

The authors did not receive any funds, grants, or other support.

\section{Conflict of interest}

The authors have no conflicts of interest to declare that are relevant to the content of this article.

\section{Authors' contributions}

First author: Writing original draft, developing methodology, carrying-out formal analysis.

Second author: Research and methodology ideas, review of mathematical and analysis parts \& editing, Third author: Supervision, writing, review \& editing.

\section{References}

Akram M, Ilyas F, Garg H (2020) Multi-criteria group decision making based on electre I method in pythagorean fuzzy information. Soft Computing 24(5):3425-3453

Bouyssou D (1999) Using DEA as a tool for MCDM: some remarks. Journal of the Operational Research Society 50(9):974-978

Büyüközkan G, Göçer F, Karabulut Y (2019) A new group decision making approach with IF AHP and IF VIKOR for selecting hazardous waste carriers. Measurement 134:66-82

Çalı S, Balaman ŞY (2019) A novel outranking based multi criteria group decision making methodology integrating ELECTRE and VIKOR under intuitionistic fuzzy environment. Expert Systems with Applications 119:36-50

Charnes A, Cooper WW, Rhodes E (1978) Measuring the efficiency of decision making units. European Journal of Operational Research 2(6):429-444

Dammak F, Baccour L, Alimi AM (2020) A new ranking method for TOPSIS and VIKOR under interval valued intuitionistic fuzzy sets and possibility measures. Journal of Intelligent and Fuzzy Systems (Preprint):1-11

Dong Q, Cooper O (2016) A peer-to-peer dynamic adaptive consensus reaching model for the group AHP decision making. European Journal of Operational Research 250(2):521-530

Doyle J, Green R (1994) Efficiency and cross-efficiency in DEA: Derivations, meanings and uses. Journal of the Operational Research Society 45(5):567-578

Dursun M, Karsak EE (2013) A QFD-based fuzzy MCDM approach for supplier selection. Applied Mathematical Modelling 37(8):5864-5875

Ebrahimnejad A, Tavana M, Nasseri SH, Gholami O (2019) A new method for solving dual DEA problems with fuzzy stochastic data. International Journal of Information Technology and Decision Making 18(01):147-170

Ehrgott M, Klamroth K, Schwehm C (2004) An MCDM approach to portfolio optimization. European Journal of Operational Research 155(3):752-770 
Farhadinia B, Xu Z (2020) A novel distance-based multiple attribute decision-making with hesitant fuzzy sets. Soft Computing 24:5005-5017

Farrell MJ (1957) The measurement of productive efficiency. Journal of the Royal Statistical Society: Series A (General) 120(3):253-281

Gao K, Sun L, Yang Y, Meng F, Qu X (2021) Cumulative prospect theory coupled with multi-attribute decision making for modeling travel behavior. Transportation Research Part A: Policy and Practice 148:121

Garg H (2018) Linguistic Pythagorean fuzzy sets and its applications in multiattribute decision-making process. International Journal of Intelligent Systems 33(6):1234-1263

Gazioğlu Ş, Çalışkan N (2011) Cumulative prospect theory challenges traditional expected utility theory. Applied Financial Economics 21(21):1581-1586

Geng X, Qiu H, Gong X (2017) An extended 2-tuple linguistic dea for solving MAGDM problems considering the influence relationships among attributes. Computers and Industrial Engineering 112:135-146

Guo S, Zhao H (2015) Optimal site selection of electric vehicle charging station by using fuzzy TOPSIS based on sustainability perspective. Applied Energy 158:390-402

Harel A, Francis JC, Harpaz G (2018) Alternative utility functions: review, analysis and comparison. Review of Quantitative Finance and Accounting 51(3):785811

Herrera F, Martínez L (2000) A 2-tuple fuzzy linguistic representation model for computing with words. IEEE Transactions on Fuzzy Systems 8(6):746-752

Herrera F, Herrera-Viedma E, Martínez L (2008) A fuzzy linguistic methodology to deal with unbalanced linguistic term sets. IEEE Transactions on Fuzzy Systems 16(2):354-370

Ju Y (2014) A new method for multiple criteria group decision making with incomplete weight information under linguistic environment. Applied Mathematical Modelling 38(21-22):5256-5268

Kahneman D, Tversky A (1979) Prospect theory: An analysis of decision under risk. Econometrica 47(2):263-292
Koksalmis E, Kabak Ö (2019) Deriving decision makers' weights in group decision making: An overview of objective methods. Information Fusion 49:146-160

Li H, Wang W, Fan L, Li Q, Chen X (2020) A novel hybrid MCDM model for machine tool selection using fuzzy DEMATEL, entropy weighting and later defuzzification VIKOR. Applied Soft Computing 91:106-207

Liang D, Xu Z (2017) The new extension of topsis method for multiple criteria decision making with hesitant Pythagorean fuzzy sets. Applied Soft Computing 60:167-179

Liu HC, You JX, Lu C, Chen YZ (2015) Evaluating health-care waste treatment technologies using a hybrid multi-criteria decision making model. Renewable and Sustainable Energy Reviews 41:932-942

Liu Hh, Song Yy, Yang Gl (2019) Cross-efficiency evaluation in data envelopment analysis based on prospect theory. European Journal of Operational Research 273(1):364-375

Liu J, Yang JB, Wang J, SIIII HS, Wang YM (2004) Fuzzy rule-based evidential reasoning approach for safety analysis. International Journal of General Systems 33(2-3):183-204

Liu W, Li L (2019) Emergency decision-making combining cumulative prospect theory and group decisionmaking. Granular Computing 4(1):39-52

Liu Y, Fan ZP, Zhang Y (2014) Risk decision analysis in emergency response: A method based on cumulative prospect theory. Computers and Operations Research 42:75-82

Malhotra T, Gupta A (2020a) A new 2-tuple linguistic approach for unbalanced linguistic term sets. IEEE Transactions on Fuzzy Systems https://DOI: 10.1109/TFUZZ.2020.2994987

Malhotra T, Gupta A (2020b) A systematic review of developments in the 2-tuple linguistic model and its applications in decision analysis. Soft Computing https://doi.org/10.1007/s00500-020-05031-2

Martínez L, Herrera F (2012) An overview on the 2tuple linguistic model for computing with words in decision making: extensions, applications and challenges. Information Sciences 207:1-18 
Papapostolou A, Karakosta C, Doukas H (2017) Analysis of policy scenarios for achieving renewable energy sources targets: A fuzzy TOPSIS approach. Energy and Environment 28(1-2):88-109

Qi X, Yu X, Wang L, Liao X, Zhang S (2019) PROMETHEE for prioritized criteria. Soft Computing 23(22):11419-11432

Qi XG, Guo B (2014) Determining common weights in data envelopment analysis with Shannon's entropy. Entropy 16(12):6394-6414

Ramanathan R, Ganesh L (1994) Group preference aggregation methods employed in AHP: An evaluation and an intrinsic process for deriving members weightages. European Journal of Operational Research 79(2):249-265

Saaty T (1980) The Analytic Hierarchy Process. McGraw-Hill, New York

Sexton TR, Silkman RH, Hogan AJ (1986) Data envelopment analysis: Critique and extensions. New Directions for Program Evaluation 1986(32):73-105

Shannon CE (1948) A mathematical theory of communication. The Bell System Technical Journal 27(3):379-423

Soleimani-Damaneh M, Zarepisheh M (2009) Shannon's entropy for combining the efficiency results of different DEA models: Method and application. Expert Systems with Applications 36(3):5146-5150

Song L, Liu F (2018) An improvement in DEA crossefficiency aggregation based on the Shannon entropy. International Transactions in Operational Research 25(2):705-714

Sun B, Qi C, Ma W, Wang T, Zhang L, Jiang C (2020) Variable precision diversified attribute multigranulation fuzzy rough set-based multi-attribute group decision making problems. Computers and Industrial Engineering 142:331-339

Tian C, Peng J (2020) An integrated picture fuzzy ANPTODIM multi-criteria decision-making approach for tourism attraction recommendation. Technological and Economic Development of Economy 26(2):331354

Torra V (2000) Knowledge-based validation: Synthesis of diagnoses through synthesis of relations. Fuzzy Sets and Systems 113(2):267-276
Tversky A, Kahneman D (1992) Advances in prospect theory: Cumulative representation of uncertainty. Journal of Risk and Uncertainty 5(4):297-323

Wan SP, Wang F, Lin LL, Dong JY (2016) Some new generalized aggregation operators for triangular intuitionistic fuzzy numbers and application to multiattribute group decision making. Computers and Industrial Engineering 93:286-301

Wang L, Li L, Hong N (2016) Entropy cross-efficiency model for decision making units with interval data. Entropy 18(10):358

Wang T, Li H, Zhang L, Zhou X, Huang B (2020) A threeway decision model based on cumulative prospect theory. Information Sciences 519:74-92

Wu J, Sun J, Liang L (2012) DEA cross-efficiency aggregation method based upon Shannon entropy. International Journal of Production Research 50(23):67266736

Wu Z, Tu J (2021) Managing transitivity and consistency of preferences in AHP group decision making based on minimum modifications. Information $\mathrm{Fu}-$ sion 67:125-135

Xie Q, Dai Q, Li Y, Jiang A (2014) Increasing the discriminatory power of DEA using Shannon's entropy. Entropy 16(3):1571-1585

Yayla AY, Oztekin A, Gumus AT, Gunasekaran A (2015) A hybrid data analytic methodology for 3PL transportation provider evaluation using fuzzy multicriteria decision making. International Journal of Production Research 53(20):6097-6113

Yue Z (2013) Group decision making with multiattribute interval data. Information Fusion 14(4):551-561

Zhao M, Wei G, Wei C, Wu J (2021) Improved TODIM method for intuitionistic fuzzy MAGDM based on cumulative prospect theory and its application on stock investment selection. International Journal of Machine Learning and Cybernetics 12(3):891-901 